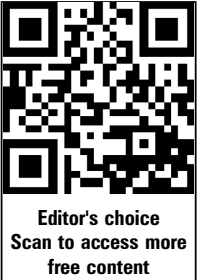

${ }^{1}$ Department of Public Health, University of Helsinki, Helsinki, Finland

${ }^{2}$ Finnish Institute of

Occupational Health, Helsinki, Finland

\section{Correspondence to}

Dr E Lahelma, Department of Public Health, University of Helsinki, P.O. Box 41, Helsinki 00014, Finland; eero.lahelma@helsinki.fi

Received 1 July 2014 Revised 28 October 2014 Accepted 30 October 2014 Published Online First 14 November 2014

\title{
Common mental disorders and cause-specific disability retirement
}

\author{
E Lahelma, ${ }^{1}$ O Pietiläinen, ${ }^{1}$ O Rahkonen, ${ }^{1}$ T Lallukka ${ }^{1,2}$
}

ABSTRACT
Objectives Common mental disorders are prevalent among employees and may cause work disability. We aimed to examine the association between common mental disorders and disability retirement, with an emphasis on the severity of disorders and diagnostic causes for retirement.

Methods Our data were derived from the Helsinki Health Study cohort on the staff of the City of Helsinki, Finland. The baseline mail surveys were made in 20002002 among employees reaching ages 40, 45, 50, 55 and 60 in each year $(n=8960$, response rate $67 \%, 80 \%$ women). Disability retirement events from national registers ( $n=628$ ) were followed up by the end of 2010 and linked to the baseline data. After exclusions, the number of participants was 6525. Common mental disorders were measured by the General Health Questionnaire 12-item version (GHQ-12). Covariates at baseline included sociodemographic, work-related and health-related factors. Hazard ratios (HR) and 95\% Cls were calculated using Cox proportional hazards models. Results Common mental disorders showed a graded association with disability retirement. For disability retirement due to any diagnostic cause, the fully adjusted HR for the GHQ-12 score 7-12 was 2.16, 95\% $\mathrm{Cl} 1.63$ to 2.85 . For disability retirement due to mental disorders the corresponding HR was $7.46,95 \% \mathrm{Cl} 4.46$ to 12.49 . For disability retirement due to musculoskeletal diseases, the association was weaker and did not survive all adjustments.

Conclusions Common mental disorders are an important antecedent of disability retirement in general and due to mental disorders in particular. Successful measures against common mental disorders may prevent disability retirement due to mental disorders.

\section{INTRODUCTION}

Mental disorders are increasingly important public health problems worldwide. ${ }^{1}$ Symptoms of depression, anxiety and lack of self-esteem, often called common mental disorders, affect both the general and employed populations. Evidence from different countries suggests that about one-fourth or onefifth among the middle-aged employees suffer from common mental disorders. ${ }^{2-6}$ These disorders impair people's quality of life, functioning and work ability, ${ }^{78}$ leading even to premature exit from work life through disability retirement. ${ }^{9-12}$ In the Organisation for Economic Cooperation and Development (OECD) countries, mental disorders and musculoskeletal diseases are the dominant diagnostic causes of disability retirement. ${ }^{13}$

\section{What this paper adds}

- Common mental disorders have shown associations with disability retirement.

- The contribution of the severity of general common mental disorders to disability retirement due to various diagnostic causes is poorly understood.

- The association between common mental disorders and disability retirement followed a gradient.

- The association was particularly strong for most severe common mental disorders and for disability retirement due to mental disorders.

In Finland, mental disorders account for about $40 \%$, while musculoskeletal diseases account for about $30 \%$ of the stock of all disability retirements. $^{14}$

Work disability emerges as a mismatch between health status and work environment factors, which in serious cases may result in subsequent disability retirement. ${ }^{15}$ We are thus dealing with a major transition in an employee's life course that has consequences for the employee, the workplace as well as the labour market and the economy. These issues are particularly topical as populations are ageing, life expectancy is increasing and longer work careers are expected. ${ }^{16} 17$ The burden of work disability is high in European countries where 5-10\% of the working aged are on disability retirement ${ }^{13}$; in Finland, the current figure is $7 \% .{ }^{14}$ It is important to be able to tackle disability retirement, and for this purpose we need a better understanding of the reasons for disability retirement. The particular focus of this study is on the contribution of common mental disorders to disability retirement. This focus is justified regarding the high significance of mental health in work-life in general and in the processes leading to work disability and early retirement in particular.

Some previous studies have examined the associations between common mental disorders and disability retirement. A Danish study among the general population found an association between symptoms of depression and subsequent disability retirement. ${ }^{10}$ Studies from Finland have also shown a similar association for depression and general common mental disorders among farmers, ${ }^{18}$ elderly men ${ }^{19}$ and in the adult population. ${ }^{20}$ The associations have followed a gradient, with more severe common mental disorders being more 
strongly associated with disability retirement due to any cause. Such a gradient was strong for mental diagnostic causes of retirement, but was also observed for musculoskeletal and cardiovascular causes ${ }^{19}$ as well as somatic causes in general. ${ }^{9} 12$ These associations have remained after considering sociodemographic, work-related and health-related covariates.

Studies from Norway ${ }^{921}$ and Finland ${ }^{20} 22$ have examined the association between clinically significant depression and anxiety as well as their comorbidity and disability retirement due to any diagnostic cause. Depression and anxiety separately were associated with disability retirement, but comorbid conditions showed the strongest associations. The associations remained after considering sociodemographic, health-related and workrelated factors.

Most previous studies have placed more emphasis on more severe clinically significant depression and anxiety. However, a study from Stockholm, Sweden ${ }^{12}$ examined common mental disorders using the General Health Questionnaire (GHQ), ${ }^{23} 24$ an instrument widely used in screening and epidemiological studies. The GHQ gives a quantitative score of general common mental disorders and allows the severity to be examined. The study from Stockholm showed a strong and consistent gradient by the severity of common mental disorders even after considering sociodemographic and health-related covariates. ${ }^{12}$ The gradient was very steep for disability retirement due to diagnosed mental disorders but was also observed for somatic diagnoses in general.

A number of previous studies have examined the risk of common mental disorders and clinically significant mental problems for diagnosis-specific disability retirement. ${ }^{9} 12 \quad \begin{array}{llll}12 & 21 & 22 & 25\end{array}$ They confirm that prior depression and anxiety as well as their comorbidity contribute to subsequent disability retirement due to any cause as well as mental disorders, but possibly also due to somatic causes, such as musculoskeletal and cardiovascular diseases. The same concerns general common mental disorders, but their contribution to disability retirement remains inadequately understood as studies are rare. More evidence is needed for the mental risk factors for disability retirement due to mental and even somatic causes.

While common mental disorders undermine functioning and work ability, their importance may remain partly hidden. Healthcare plays a key role in the assessment of work ability and medical examination is a necessary condition for determining work disability and awarding disability pension. Common mental disorders may remain unrecognised and unassessed for work disability if people do not seek help. Even in healthcare, patients and professionals may neglect common mental disorders, regard them as trivial or may not be conscious of them. Such instances might lead to underestimation of the importance of common mental disorders to the process eventually leading to disability retirement. ${ }^{12} 21$

Our study aimed to contribute to the understanding of the association between common mental disorders and subsequent disability retirement. The specific aims were to investigate the association between general common mental disorders and disability retirement (1) considering the severity of common mental disorders, (2) exploring disability retirement due to any diagnostic cause as well as mental disorders and musculoskeletal diseases and (3) examining sociodemographic, health-related and work-related factors as covariates.

\section{DATA AND METHODS}

\section{Baseline data}

The data for this study were derived from the Helsinki Health Study cohort on the staff of the City of Helsinki. The City of
Helsinki is the largest employer in Finland, with almost 40000 employees. It provides basic services, including health and social welfare, education and culture, technical maintenance, public transportation and general administration. The staff includes hundreds of blue-collar and white-collar occupations. ${ }^{26}$

The baseline surveys were collected in 2000, 2001 and 2002 among employees who reached the ages of 40, 45, 50, 55 and 60 in each year. The sample included 13344 employees to whom mail questionnaires were sent. At baseline, 8960 employees participated (response rate 67\%, 80\% women). The gender distribution corresponds to the staff of the City of Helsinki and the Finnish municipal sector. Non-response analyses showed that overall the baseline respondents represented the target population, with men, younger employees, manual workers and those with prolonged sick sickness absence being slightly under-represented. ${ }^{26}$

\section{Disability retirement}

Data on disability retirement were obtained from the registers of the Finnish Centre for Pensions, providing complete information of all retirement events. Disability retirement events were followed up by the end of 2010 and individually linked to the baseline survey data for those who had provided written consent for data linkage (74\%). Participants who during the follow-up retired based on age $(n=1170)$, reached 63 years $(n=204)$ or died $(n=49)$ were censored. In the Finnish retirement scheme, pensions granted after the age of 63 years are no longer based on disability but on age alone. After exclusions, the number of participants was 6525. Non-response analyses also examined the effect of consenting to data linkage and showed that bias by sociodemographics and sickness absence was minor. ${ }^{26}$

Medically confirmed diagnosis is required for granting disability pension in the Finnish pension scheme, and the main disability retirement diagnoses (ICD-10) were derived from the register data. Disability retirement events due to any diagnostic cause amounted to 628. The largest diagnostic groups, that is, mental disorders (ICD-10 F00-F99, $\mathrm{n}=166$ ) and musculoskeletal diseases (ICD-10 M00-M99, $n=271$ ), were examined separately.

\section{Common mental disorders}

Common mental disorders were derived from the baseline surveys and measured by the GHQ 12-item version (GHQ-12), which is a reliable and well-validated inventory of symptoms of minor mental health problems. Common mental disorders as indicated by GHQ-12 refer to general and context-free affective and non-psychotic problems in the recent past, such as symptoms of depression, anxiety and self-esteem. It gives a quantitative estimate along one dimension, the scores ranging from 0 to 12 . Cronbach's $\alpha$ coefficient for internal consistency in these data was 0.91 . GHQ-12 scores are distributed throughout populations to varying degrees, and common dichotomisations include $1 / 2$, $2 / 3$ and $3 / 4$. To be able to examine grades in the severity of common mental disorders and to compare these to prior studies, we classified the scores to $0-1,2,3-6$ and $7-12$. Scores $0-1$ were used as the reference category in the analyses. Validation studies show that GHQ-12 is predictive of the need for treatment and onset of more severe mental disorders. ${ }^{23} 242728$

\section{Covariates}

Sociodemographic covariates included age and gender; marital status categorised into married, unmarried and divorced/ widowed; and occupational class derived from the employer's 
personnel register categorised into managers and professionals, semi-professionals, routine non-manual and manual workers. Following our previous procedures, three domains of working conditions were included based on self-reports. ${ }^{29}$ 30 Work arrangements included working $40 \mathrm{~h}$ or more per week versus less, shift work versus normal working hours, and permanent versus temporary work contract. Physical working conditions included physical workload, hazardous exposures and computer work dichotomised by the upper quartile of adversity. ${ }^{31}$ Psychosocial working conditions included Karasek's ${ }^{32}$ job strain and Sarason's ${ }^{33}$ social support at work dichotomised closest to the upper quartile of adversity.

Health behaviours and body mass index (BMI) were included following our previous procedures. ${ }^{34}$ Smoking was dichotomised into current daily smokers and non-smokers (including ex-smokers). Drinking for men was regarded as heavy when estimated consumption exceeded $280 \mathrm{~g}$ of pure alcohol per week, and for women when consumption exceeded $140 \mathrm{~g}$ following the Finnish Current Care Guidelines. ${ }^{35}$ Physical activity at leisure time was measured by four questions and metabolic equivalents (MET) were calculated. Having fewer than 14 MET hours per week was classified as being inactive and others as active. BMI was calculated from self-reported height and weight $\left(\mathrm{kg} / \mathrm{m}^{2}\right)$ and a BMI of $30 \mathrm{~kg} / \mathrm{m}^{2}$ or more indicated obesity.

\section{Statistical methods}

Multiple imputation for missing values on the studied variables was conducted. ${ }^{36}$ The imputation process was used to create 10 data sets with missing values replaced by imputed values. The analyses were then run on each imputed data set and the results were combined by taking averages of the coefficients and adjusting SEs for the imputation. The data were assumed to be missing at random.

Associations between common mental disorders and disability retirement were examined using Cox proportional hazards models. Gender differences were tested by fitting interactions. No interactions were found, and men and women were pooled in the analyses. Models were fitted to yield HRs and their 95\% CIs, indicating the risk of disability retirement among those suffering from common mental disorders at varying levels while adjusting for covariates. Model 1 adjusted for age and gender. Model 2 included model 1 and adjusted additionally for marital status, socioeconomic position and social support. Model 3 included model 1 and adjusted additionally for working conditions. Model 4 included model 1 and adjusted additionally for health behaviours and BMI. Model 5 included model 1 and adjusted additionally for limiting long-standing illness. Model 6 was a fully adjusted model including all variables simultaneously. All analyses were made using R statistical software V.2.13.0.

\section{RESULTS}

At baseline, 68\% scored 0-1 in GHQ-12, 7\% scored 2, 13\% scored 3-6 and 11\% scored 7-12 (table 1). The prevalence of scoring 3-12 was 24\%, 26\% for women and 23\% for men.

In the age-adjusted and gender-adjusted model 1, the association between common mental disorders and all-cause disability retirement was clear and followed a gradient (table 2). The HR for scoring 2 in GHQ-12 was 1.43 (95\% CI 1.05 to 1.95 ), for scoring 3-6 the HR was 1.67 (95\% CI 1.34 to 2.08), and for scoring 7-12 the HR was 3.20 (95\% CI 2.64 to 3.87 ). Adjusting for limiting long-standing illness in model 5 attenuated the association by about a third, but a graded association remained $(\mathrm{HR}=2.24,95 \% \mathrm{CI} 1.83$ to 2.75$)$. Otherwise, the effects of adjustments were minor.

There was a stronger association between common mental disorders and disability retirement due to diagnosed mental disorders (table 3). Adjusting for age and gender in model 1, the HR for scoring 2 in GHQ-12 was 2.05 (95\% CI 1.10 to 3.81 ), for scoring 3-6 the HR was 2.06 (95\% CI 1.27 to 3.33 ), and for scoring 7-12 the HR was 8.20 (95\% CI 5.81 to 11.58). Thus, this association also tended to be graded, but there was an additional threshold effect as the HR for the highest GHQ-12 scores (7-12) was particularly strong compared to lower GHQ-12 scores. Adjusting for limiting long-standing illness in model 5 attenuated the association by 14\%, but it remained strong $(\mathrm{HR}=7.06,95 \% \mathrm{CI} 4.83$ to 10.31$)$. Otherwise, the effects of adjustments were minor.

The association between common mental disorders and disability retirement due to musculoskeletal diseases was weaker than that for all causes but also followed a gradient when adjusted for age and gender in model 1 (table 4). The HR for scoring 2 in GHQ-12 was 1.39 (95\% CI 0.88 to 2.19), for scoring 3-6 the HR was 1.66 (95\% CI 1.20 to 2.28$)$, and for scoring 7-12 the HR was 1.99 (95\% CI 1.44 to 2.75$)$. The association remained after adjusting for sociodemographics in model 2. Adjusting for work factors in model 3, the association attenuated by about a fifth, and this was due to physical work exposures. Adjusting for health behaviours and BMI in model 4, the association also attenuated by about a fifth, and this was due to smoking and BMI. Adjusting for limiting long-standing illness in model 5 , the association attenuated further and did not reach statistical significance.

\section{DISCUSSION}

This study sought to find out whether general common mental disorders, varying in severity, are associated with subsequent disability retirement due to any diagnostic cause and due to two main diagnostic causes, that is, mental disorders and musculoskeletal diseases.

The main findings of the study can be summarised as follows. First, common mental disorders showed a graded association

Table 1 Distribution of common mental disorders (GHQ-12 score) and disability retirement events by diagnosis

\begin{tabular}{|c|c|c|c|c|c|c|c|c|}
\hline \multirow[b]{2}{*}{ GHQ-12 score } & \multicolumn{2}{|c|}{ Common mental disorders } & \multicolumn{2}{|c|}{ All causes } & \multicolumn{2}{|c|}{ Mental causes } & \multicolumn{2}{|c|}{ Musculoskeletal causes } \\
\hline & $\mathbf{N}$ & Per cent & $\mathrm{N}$ & Per cent & $\mathrm{N}$ & Per cent & $\mathrm{N}$ & Per cent \\
\hline $0-1$ & 4435 & 68 & 319 & 51 & 58 & 35 & 152 & 56 \\
\hline 2 & 447 & 7 & 46 & 7 & 12 & 7 & 21 & 8 \\
\hline $3-6$ & 870 & 13 & 104 & 17 & 23 & 14 & 50 & 18 \\
\hline $7-12$ & 733 & 11 & 159 & 25 & 73 & 44 & 48 & 18 \\
\hline All & 6485 & 100 & 628 & 100 & 166 & 100 & 271 & 100 \\
\hline
\end{tabular}


Table 2 Common mental disorders (GHQ-12 score) and all-cause disability retirement

\begin{tabular}{|c|c|c|c|c|c|c|c|c|c|c|c|c|}
\hline \multirow[b]{2}{*}{ GHQ-12 score } & \multicolumn{2}{|c|}{ Model 1} & \multicolumn{2}{|c|}{ Model 2} & \multicolumn{2}{|c|}{ Model 3} & \multicolumn{2}{|c|}{ Model 4} & \multicolumn{2}{|c|}{ Model 5} & \multicolumn{2}{|c|}{ Model 6} \\
\hline & HR & $95 \% \mathrm{Cl}$ & HR & $95 \% \mathrm{Cl}$ & HR & $95 \% \mathrm{Cl}$ & HR & $95 \% \mathrm{Cl}$ & HR & $95 \% \mathrm{Cl}$ & HR & $95 \% \mathrm{Cl}$ \\
\hline $0-1$ & 1.00 & & 1.00 & & 1.00 & & 1.00 & & 1.00 & & 1.00 & \\
\hline 2 & 1.43 & 1.05 to 1.95 & 1.53 & 1.12 to 2.09 & 1.25 & 0.86 to 1.81 & 1.27 & 0.87 to 1.86 & 1.16 & 0.84 to 1.59 & 1.08 & 0.70 to 1.67 \\
\hline $3-6$ & 1.67 & 1.34 to 2.08 & 1.80 & 1.44 to 2.25 & 1.75 & 1.36 to 2.24 & 1.70 & 1.33 to 2.19 & 1.28 & 1.02 to 1.62 & 1.56 & 1.16 to 2.09 \\
\hline $7-12$ & 3.20 & 2.64 to 3.87 & 3.38 & 2.78 to 4.10 & 2.97 & 2.36 to 3.73 & 2.86 & 2.29 to 3.57 & 2.24 & 1.83 to 2.75 & 2.16 & 1.63 to 2.85 \\
\hline
\end{tabular}

HRs and their $95 \%$ Cls from Cox regression analysis adjusted for covariates.

Model $1=$ Age and gender.

Model 2=Model 1+marital status+social support+socioeconomic position.

Model $3=$ Model $1+$ job strain+physical work exposures.

Model 4=Model 1+health behaviours+BMI.

Model $5=$ Model $1+$ limiting long-standing illness.

Model $6=$ Fully adjusted.

BMI, body mass index; GHQ, General Health Questionnaire.

with disability retirement due to any diagnostic cause. This association attenuated by about a third after considering prior limiting long-standing illness. Second, common mental disorders showed a strong association with disability retirement due to mental disorders. There was a threshold implying that for those with most severe common mental disorders the risk of disability retirement due to mental disorders was particularly high. Adjusting for limiting long-standing illness attenuated the association but less than for other diagnostic causes. Third, even for musculoskeletal diseases, an association between common mental disorders and disability retirement was suggested, but this association did not survive the adjustment for limiting longstanding illness.

\section{Interpretation and comparison}

Work disability and subsequent disability retirement take place as a function of employee health status and the requirements of work environment. ${ }^{15} 30$ In this study, the focus was on health factors, in particular mental health as indicted by general common mental disorders.

Common mental disorders are prevalent among employees, and in our study a quarter reported a GHQ-12 score of 3 or more, an often used dichotomisation for having common mental disorders. ${ }^{23} 2428$ Similar prevalence rates have been observed in other studies using the same measurement. ${ }^{5}{ }^{6}$ Thus, common mental disorders affect functioning and work ability among large segments of employees. The association between common mental disorders and subsequent disability retirement was clear and consistent in our study. The risk of disability retirement was gradually stronger the more severe the common mental disorders were, which is in accordance with previous studies. ${ }^{12} 1819$ This implies that an excess risk can be seen already at low levels of common mental disorders and that the risk is particularly strong for those with the most severe common mental disorders.

The other side of work disability, that is, work environment indicated by work stress and physical exposures, contributes to disability retirement, ${ }^{30}$ but their effects on the association between common mental disorders and disability retirement were minor in this study. Physical workload explained a part of the association between common mental disorders and disability retirement, in particular due to musculoskeletal disorders. In another Finnish study, psychosocial job strain was associated with disability retirement due to musculoskeletal disorders but not due to depression. ${ }^{25}$

Among further covariates, the effects of sociodemographics and social support on the associations studied by us were negligible. These factors are known to affect common mental disorders. ${ }^{37}$ However, work disability is based on a comprehensive assessment of a person's resources to continue to work, and a diagnosed disease is in practice a necessary condition for being granted a disability pension award in the Finnish retirement system. ${ }^{14}{ }^{15}$ The importance of health-related factors was also apparent in our findings. Health behaviours attenuated the association between common mental disorders and disability retirement to some extent. The attenuation due to limiting long-standing illness was almost a third, suggesting that common mental disorders

Table 3 Common mental disorders (GHQ-12 score) and disability retirement due to mental causes

\begin{tabular}{|c|c|c|c|c|c|c|c|c|c|c|c|c|}
\hline \multirow[b]{2}{*}{ GHQ-12 score } & \multicolumn{2}{|c|}{ Model 1} & \multicolumn{2}{|c|}{ Model 2} & \multicolumn{2}{|c|}{ Model 3} & \multicolumn{2}{|c|}{ Model 4} & \multicolumn{2}{|c|}{ Model 5} & \multicolumn{2}{|c|}{ Model 6} \\
\hline & HR & $95 \% \mathrm{Cl}$ & HR & $95 \% \mathrm{Cl}$ & HR & $95 \% \mathrm{Cl}$ & HR & $95 \% \mathrm{Cl}$ & HR & $95 \% \mathrm{Cl}$ & HR & $95 \% \mathrm{Cl}$ \\
\hline $0-1$ & 1.00 & & 1.00 & & 1.00 & & 1.00 & & 1.00 & & 1.00 & \\
\hline 2 & 2.05 & 1.10 to 3.81 & 2.13 & 1.14 to 3.98 & 1.72 & 0.81 to 3.66 & 1.62 & 0.72 to 3.61 & 1.88 & 0.97 to 3.62 & 1.57 & 0.60 to 4.10 \\
\hline $3-6$ & 2.06 & 1.27 to 3.33 & 2.02 & 1.23 to 3.30 & 2.20 & 1.29 to 3.77 & 2.07 & 1.19 to 3.60 & 1.93 & 1.16 to 3.22 & 2.26 & 1.18 to 4.32 \\
\hline $7-12$ & 8.20 & 5.81 to 11.58 & 7.98 & 5.62 to 11.35 & 8.07 & 5.36 to 12.17 & 7.66 & 5.11 to 11.49 & 7.06 & 4.83 to 10.31 & 7.46 & 4.46 to 12.49 \\
\hline
\end{tabular}

HRs and their $95 \% \mathrm{Cls}$ from Cox regression analysis adjusted for covariates.

Model $1=$ Age and gender.

Model 2=Model 1+marital status+social support+socioeconomic position

Model 3=Model $1+$ job strain+physical work exposures.

Model $4=$ Model $1+$ health behaviours+BMI.

Model $5=$ Model $1+$ limiting long-standing illness.

Model $6=$ Fully adjusted.

BMI, body mass index; GHQ, General Health Questionnaire. 
Table 4 Common mental disorders (GHQ-12 score) and disability retirement due to musculoskeletal causes

\begin{tabular}{|c|c|c|c|c|c|c|c|c|c|c|c|c|}
\hline \multirow[b]{2}{*}{ GHQ-12 score } & \multicolumn{2}{|c|}{ Model 1} & \multicolumn{2}{|c|}{ Model 2} & \multicolumn{2}{|c|}{ Model 3} & \multicolumn{2}{|c|}{ Model 4} & \multicolumn{2}{|c|}{ Model 5} & \multicolumn{2}{|c|}{ Model 6} \\
\hline & $\mathrm{HR}$ & $95 \% \mathrm{Cl}$ & $\mathrm{HR}$ & $95 \% \mathrm{Cl}$ & HR & $95 \% \mathrm{Cl}$ & $\mathrm{HR}$ & $95 \% \mathrm{Cl}$ & HR & $95 \% \mathrm{Cl}$ & HR & $95 \% \mathrm{Cl}$ \\
\hline $0-1$ & 1.00 & & 1.00 & & 1.00 & & 1.00 & & 1.00 & & 1.00 & \\
\hline 2 & 1.39 & 0.88 to 2.19 & 1.53 & 0.97 to 2.42 & 0.94 & 0.52 to 1.71 & 1.19 & 0.67 to 2.12 & 1.03 & 0.65 to 1.66 & 0.76 & 0.38 to 1.52 \\
\hline $3-6$ & 1.66 & 1.20 to 2.28 & 1.91 & 1.39 to 2.64 & 1.66 & 1.14 to 2.40 & 1.78 & 1.24 to 2.55 & 1.15 & 0.82 to 1.61 & 1.44 & 0.92 to 2.24 \\
\hline $7-12$ & 1.99 & 1.44 to 2.75 & 2.24 & 1.61 to 3.11 & 1.57 & 1.05 to 2.35 & 1.66 & 1.12 to 2.46 & 1.21 & 0.85 to 1.72 & 0.85 & 0.50 to 1.45 \\
\hline
\end{tabular}

HRs and their $95 \% \mathrm{Cls}$ from Cox regression analysis adjusted for covariates. Model $1=$ Age and gender.

Model 2=Model 1+marital status+social support+socioeconomic position.

Model $3=$ Model $1+$ job strain+physical work exposures.

Model 4=Model 1+health behaviours+BMI.

Model $5=$ Model $1+$ limiting long-standing illness.

Model $6=$ Fully adjusted.

BMI, body mass index; GHQ, General Health Questionnaire.

are partly related to chronic somatic illnesses, ${ }^{38}$ and possibly also to mental disorders. Nevertheless, even after full adjustment, common mental disorders remained associated in a graded way with disability retirement.

The focus of our study was on general and relatively mild common mental disorders. One previous study from Sweden also examined grades of general common mental disorders measured by GHQ-12 as antecedents of disability retirement. ${ }^{12}$ Our results were very similar to that study with a strong and graded association between common mental disorders and disability retirement due to any cause which remained even after adjustments. In sensitivity analyses, we used various graded categorisations of GHQ-12. However, the associations and their shape remained. For all cause retirement, the association was linear and for mental causes it was particularly strong for severe GHQ-12 scores of 712. Several studies have focused on depression and anxiety, elicited by clinical inventories, for example, the Composite International Diagnostic Interview (CIDI). Such inventories allow for the Diagnostic and Statistical Manual of Mental Disorders (DSM-IV) diagnoses and reflect less prevalent but more severe problems than GHQ-12.9 10 19-22 39 The associations between depression and anxiety and all-cause disability retirement have been broadly similar to ours before and after adjustments.

Compared to disability retirement due to any cause, the diagnostic causes affected our results. Common mental disorders were a very strong risk factor for disability retirement due to mental disorders. In sensitivity analyses, we also subtracted retirements due to mental causes from all causes. This resulted in a third weaker association between common mental disorders and disability retirement, and the association survived all adjustments. The risk of common mental disorders for retirement due to mental causes was also graded, but there was a threshold effect showing that the $11 \%$ with most severe common mental disorders, that is, a GHQ-12 score of 7-12, showed a fourfold risk of disability retirement due to mental disorders compared to those with lower scores. This has been observed in an earlier Swedish study in which the risk of disability retirement due to mental causes was also multifold for those with a GHQ-12 score of $8-12 .^{12}$

Even mild common mental disorders conveyed a risk of deteriorating work ability. The very high risk among those with most severe common mental disorders and clinically significant mental problems should be interpreted with caution. It is possible that severe mental problems reflect less causal and more selection effects as early or existing mental problems may already have been diagnosed and treated, contributing eventually to disability retirement. To what extent the milder forms of common mental disorders will develop into more severe ones remains an open question in our study, which did not follow-up changes in common mental disorders. It should also be noted that a third of those who over the follow-up retired due to diagnosed mental disorders lacked common mental disorders at baseline. This suggests that common mental disorders indicated by GHQ-12 should not be interpreted as leading in a deterministic way to diagnosed mental disorders, but rather as a risk factor.

Our follow-up was relatively long, maximally 10 years. In sensitivity analyses, this period was broken down to two 5-year periods to see whether the association between common mental disorders and disability retirement would fade away over time. Nevertheless, the associations also remained in the later period and survived adjustments. Some temporal attenuation took place for all-cause retirement and less so for mental causes. For musculoskeletal diseases, the association was weaker and borderline statistically significant. The temporal attenuation for retirement due to mental causes was minor and less than that in a Norwegian study. ${ }^{21}$ Overall, our results confirm that GHQ is a long-term risk factor for disability retirement in general and for retirement due to mental causes in particular. The complex pathways from common mental disorders to diagnosed and disabling mental disorders to early exclusion from work warrant further investigation.

Common mental disorders were a risk factor even for somatic causes, that is, musculoskeletal diseases leading to disability retirement. The risk, however, was weaker than that for any diagnostic cause or mental causes and did not survive adjustment for limiting long-standing illness, reflecting primarily physical health problems, ${ }^{38}$ in our case most likely chronic musculoskeletal problems. Also, previous evidence suggests that common mental disorders may be a risk factor for disability retirement due to musculoskeletal and cardiovascular diagnostic causes $^{19}$ as well as somatic causes in general, ${ }^{9-12}$ but these risks have been much lower than those for mental causes. However, disability retirement is often the result of multiple comorbid conditions, for example, mental and musculoskeletal. ${ }^{9} \quad 21 \quad 40$ The complexity of the processes leading to severe work disability and premature retirement is further exemplified by findings showing that chronic and musculoskeletal pain are associated with disability retirement due to both mental and somatic disorders, even after considering prior somatic and mental health status. ${ }^{41}{ }^{42}$ While comorbidity is most likely widespread, it is still inadequately understood and studies examining secondary retirement diagnoses are warranted. 


\section{Methodological considerations}

We used a longitudinal design to study a large employee cohort including a broad range of different occupations. An advantage was that reliable and complete register-based data with diagnostic causes of disability retirement could be used. As the number of retirement events was relatively low, our analyses were limited to mental disorders and musculoskeletal diseases.

Participation to our baseline survey was acceptable and we were able to conduct extensive non-response analyses. ${ }^{26}$ These analyses suggest that major bias in the studied associations is unlikely, but we acknowledge that the non-response remains a potential source of bias.

Our measure of common mental disorders was GHQ-12. ${ }^{23} 24$ This self-administered measure covers symptoms of minor mental health problems, but does not distinguish between subscales, for example, for depression or anxiety. GHQ-12 is a reliable and well-validated measure suitable for use in general and employee populations, but we acknowledge that self-reports are subject to potential reporting bias and clinical and diagnostic tools are also needed. Our results for GHQ-12 were supported by sensitivity analyses using the Short Form- $36^{43}$ mental component summary as a measure of common mental disorders (data not shown). In these analyses, common mental disorders were equally associated with disability retirement, with strongest associations for retirement due to mental causes.

We included several commonly used physical and psychosocial work exposures which were based on self-reports. We acknowledge that objective measurements would add to the work exposures.

Caution is needed in generalising our findings to other employment sectors and general populations since we only studied one occupational setting from the Finnish public sector. Nevertheless, our findings provided both novel and confirmatory evidence, sharpening the picture on the contribution of common mental disorders to disability retirement.

\section{CONCLUSIONS}

This study showed that common mental disorders are an important antecedent of disability retirement in general and due to mental disorders in particular. Common mental disorders cover large segments of employees who are at potential risk of subsequent work disability and exclusion from work. Thus, common mental disorders, although mostly minor, should be considered seriously in workplaces and healthcare and preventive measures should be implemented. Successful measures would most likely prevent premature loss of functioning and subsequent disability retirement, and thus help lengthen work careers.

Acknowledgements The authors thank the City of Helsinki, all members of the Helsinki Health Study group, and the Finnish Centre for Pensions for providing data on retirement.

Contributors EL drafted the manuscript. All co-authors participated in the design of the study and also commented and contributed to the drafting of the manuscript. OP carried out the data analysis. All co-authors have seen the final version and accepted it.

Funding Research grants for the Helsinki Health have been received from the Academy of Finland 1129225, 1257362, Finnish Work Environment Fund 106065, 107187, 107 281, and the Signe and Ane Gyllenberg Foundation, Helsinki, Finland.

Competing interests None.

Ethics approval Ethics approval was provided by the Ethics Committees of the Department of Public Health, University of Helsinki and the health authorities of the City of Helsinki, Finland.

Provenance and peer review Not commissioned; externally peer reviewed.
Data sharing statement The data are available on request.

\section{REFERENCES}

1 Murray C, Vos T, Lozano R, et al. Disability-adjusted life years (DALYs) for 291 diseases and injuries in 21 regions, 1990-2010: a systematic analysis for the Global Burden of Disease Study 2010. Lancet 2012;380:2197-223.

2 Harnois G, Gabriel P. Mental health and work: impact, issues and good practices. Geneva: International Labour Organisation, 2000.

3 Emslie C, Fuhrer R, Hunt $\mathrm{K}$, et al. Gender differences in mental health: evidence from three organisations. Soc Sci Med 2002;54:621-4.

4 Alonso J, Petukhova M, Vilagut $G$, et al. Days out of role due to common physical and mental conditions: results from the WHO World Mental Health surveys. Mol Psychiatry 2011;16:1234-46.

5 Head J, Stansfeld SA, Ebmeier KP, et al. Use of self-administered instruments to assess psychiatric disorders in older people: validity of the General Health Questionnaire, the Center for Epidemiologic Studies Depression Scale and the self-completion version of the revised Clinical Interview Schedule. Psychol Med 2013:43:2649-56.

6 Baxter AJ, Scott KM, Ferrari AJ, et al. Challenging the myth of an "epidemic" of common mental disorders: trends in the global prevalence of anxiety and depression between 1990 and 2010. Depress Anxiety 2014;31:506-16.

7 Stansfeld SA, Fuhrer R, Head J. Impact of common mental disorders on sickness absence in an occupational cohort study. Occup Environ Med 2011;68:408-13.

8 Rai D, Skapinakis P, Wiles $N$, et al. Common mental disorders, subthreshold symptoms and disability: longitudinal study. Br J Psychiatry 2010;197:411-12.

9 Mykletun A, Overland S, Dahl AA, et al. A population-based cohort study of the effect of common mental disorders on disability pension awards. Am J Psychiatry 2006; 163:1412-18.

10 Bültmann U, Christensen KB, Burr H, et al. Severe depressive symptoms as predictor of disability pension: a 10-year follow-up study in Denmark. Eur J Public Health 2008;18:232-4

11 Knudsen AK, Skogen JC, Harvey SB, et al. Personality disorders, common mental disorders and receipt of disability benefits: evidence from the British National Survey of Psychiatric Morbidity. Psychol Med 2012;42:2631-40.

12 Rai D, Kosidou K, Lundberg M, et al. Psychological distress and risk of long-term disability: population-based longitudinal study. J Epidemiol Community Health 2012;66:586-92.

13 OECD. Sickness disability and work. Breaking the barriers. A synthesis of findings across OECD countries. Paris: Organisation for Economic Cooperation and Development, 2010.

14 Statistical yearbook of pensioners in Finland 2012. Helsinki: Finnish Centre of Pensions and The Social Insurance Institute of Finland, 2013.

15 Ilmarinen J, Gould R, Järvikoski A, et al., eds. Diversity of work ability. In: Dimensions of work ability. Helsinki: Finnish Centre for Pensions, 2008:13-24.

16 Oeppen J, Vaupel JW. Demography. Broken limits to life expectancy. Science 2002;296:1029-31.

17 Christensen K, Doblhammer G, Rau R, et al. Ageing populations: the challenges ahead. Lancet 2009;374:1196-208.

18 Manninen P, Heliövaara M, Riihimäki $H$, et al. Does psychological distress predict disability? Int J Epidemiol 1997;26:1063-70.

19 Karpansalo M, Kauhanen J, Lakka TA, et al. Depression and early retirement: prospective population based study in middle aged men. J Epidemiol Community Health 2005;59:70-4.

20 Kaila-Kangas L, Haukka E, Miranda H, et al. Common mental and musculoskeletal disorders as predictors of disability retirement among Finns. J Affect Disord 2014;165:38-44

21 Knudsen AK, Øverland S, Aakvaag HF, et al. Common mental disorders and disability pension award: seven year follow-up of the HUSK study. J Psychosom Res 2010;69:59-67.

22 Ahola K, Virtanen M, Honkonen T, et al. Common mental disorders and subsequent work disability: a population-based Health 2000 Study. J Affect Disord 2011;134:365-72.

23 Goldberg DP. The detection of psychiatric illness by questionnaire. Maudsley monograph no 21. Oxford: Oxford University Press, 1972.

24 Goldberg DP, Gater R, Sartorius N, et al. The validity of two versions of the GHQ in the WHO study of mental illness in general health care. Psychol Med 1997;27:191-7.

25 Mäntyniemi A, Oksanen T, Salo $\mathrm{P}$, et al. Job strain and the risk of disability pension due to musculoskeletal disorders, depression or coronary heart disease: a prospective cohort study of 69,842 employees. Occup Environ Med 2012:69:574-81.

26 Lahelma $\mathrm{E}$, Aittomäki A, Laaksonen $\mathrm{M}$, et al. Cohort profile: the Helsinki Health Study. Int J Epidemiol 2013;42:722-30.

27 Warr P. Work, unemployment and mental health. Oxford: Clarendon Press, 1987.

28 Makowska Z, Merecz D, Moscicka A, et al. The validity of General Health Questionnaires, GHQ-12 and GHQ-28, in mental health studies of working people. Int J Occup Med Environ Health 2003;15:353-62. 
29 Laaksonen M, Pitkäniemi J, Rahkonen 0, et al. Work arrangements, physical working conditions, and psychosocial working conditions as risk factors for sickness absence: Bayesian analysis of prospective data. Ann Epidemiol 2010;20:332-8.

30 Lahelma $\mathrm{E}$, Laaksonen $\mathrm{M}$, Lallukka T, et al. Working conditions as risk factors for disability retirement: a longitudinal register linkage study. BMC Public Health 2012;12:309.

31 Piirainen H, Hirvonen M, Elo AL. The Work and Health Interview Study 2003. Basic Report. Helsinki: Finnish Institute of Occupational Health, 2003.

32 Karasek RA. Job demands, job decision latitude, and mental strain: implications for job redesign. Admin Sci Quart 1979;24:285-308.

33 Sarason IG, Sarason BR, Shearin EN, et al. A brief measure of social support: practical and theoretical implications. J Soc Pers Relat 1987;4:497-510.

34 Lallukka T, Laaksonen E, Martikainen $P$, et al. Associations of job strain and working overtime with adverse health behaviors and obesity: evidence from the Whitehall II Study, Helsinki Health Study and Japanese Civil Servants Study. Soc Sci Med 2008;66:1681-98.

35 Current Care Guidelines. Treatment of Alcohol Abuse. Finnish Current Care Summary. http://www.kaypahoito.fi/web/english/guidelines/guideline?id=ccs00005 (accessed 18 Jun 2014).
36 Alzola CF, Harrell FE. An Introduction to $S$ and the Hmisc and Design Libraries. 2006:1-298. Available at http://biostat.mc.vanderbilt.edu/twiki/pub/Main/RS/sintro. pdf. (accessed 18 Jun 2014).

37 Stansfeld S, Candy B. Psychosocial work environment and mental healthmeta-analytic review. Scand J Work Environ Health 2006;32:443-62.

38 Manderbacka K. How do respondents understand survey questions on ill-health? Eur J Public Health 1998;8:319-24.

39 Wedegaertner F, Arnhold-Kerri S, Sittaro NA, et al. Depression- and anxiety-related sick leave and the risk of permanent disability and mortality in the working population in Germany: a cohort study. BMC Public Health 2013;13:145.

40 OECD. Sick on the job? Myths and realities about mental health and work. Paris: Organisation for Economic Cooperation and Development, 2012.

41 Saastamoinen $\mathrm{P}$, Laaksonen $\mathrm{M}$, Kääriä S-M, et al. Pain and disability retirement: a follow up study. Pain 2012;153:526-31.

42 Øverland S, Harvey SB, Knudsen AK, et al. Widespread pain and medically certified disability pension in the Hordaland Health Study. Eur J Pain 2012;16:611-20

43 Ware JE, Kosinski M. SF-36 Physical and Mental Health Summary Scales: a Manual for Users of Version 1, 2nd edn. Lincoln: Quality-Metric, 2001. 